\title{
I Mehr als 50 Jahre Arbeitsförderungsgesetz: Die Entwicklung der deutschen Arbeitsmarktpolitik als Fortschritt oder Rückschritt?
}

\author{
Irene Dingeldey ${ }^{\star}$
}

Zusammenfassung

Das Arbeitsförderungsgesetz (AFG) von 1969 gilt als besonders progressiver Entwurf einer aktiven Arbeitsmarktpolitik in Deutschland. Anders als in der Vergangenheit blieben Würdigungen zum Jubiläum im vergangenen Jahr weitgehend aus - möglicherweise weil das AFG 1998 ins Sozialgesetzbuch III integriert und ein Paradigmenwechsel von der aktiven zur aktivierenden Arbeitsmarktpolitik vollzogen wurde. Gleichwohl nehmen wir das AFG zum Ausgangspunkt, analysieren jedoch auch die Reformen der verschiedenen Transferleistungssysteme, einschließlich der Reformen der Sozialhilfe sowie punktuell relevante Leistungen in flankierenden Politikfeldern, um die Frage nach sozialem Fortschritt in der Arbeitsmarktpolitik in verschiedenen Phasen zu beantworten. Da verschiedene internationale Organisationen jüngst Programmpapiere für eine zukunftsorientierte Arbeitsmarkt- und Sozialpolitik veröffentlichten, nutzen wir die übergreifenden Elemente, um einen Maßstab für Fortschritt oder Rückschritt zu erarbeiten. Unter dem Vorbehalt, dass die aufgezeigten arbeitsmarktpolitischen und sozialen Entwicklungen nicht ausschließlich durch die Arbeitsmarktpolitik, sondern vielfach durch sozialen und ökonomischen Wandel bedingt sind, sind Ambivalenzen hinsichtlich Fortschritt und Rückschritt zu konstatieren.

Schlagwörter: Deutschland, Arbeitsmarktpolitik, sozialer Fortschritt, Wandel

50 years after passing the employment promotion law: The development of German labour market policy as progress or regression?

Abstract

The employment promotion law (Arbeitsförderungsgesetz, AFG) from 1969 is regarded as a particularly advanced blueprint of active labour market policy in Germany. In contrast to past anniversaries appraisals in 2019 were missing - possibly because the AFG had been integrated into the Social Law Codebook III and a paradigm shift from active to activating labour market policy was performed. Nevertheless, we take the AFG as a starting point to answer the question about social progress made in different stages of labour market policy - punctually including reforms of unemployment and social benefits as well as exemplary services in related policy fields. As various international organisations recently published strategy papers on a future-oriented labour market and social policy, we create a benchmark of progress based on common elements there in. The result indicates ambivalences of progress and regress with reservation to the fact that the indicated changes are not exclusively generated by labour market policy, but often by social and economic change.

Keywords: Germany, labour market policy, social progress, change 
Neben vielen anderen Jubiläen war 2019 auch das Inkrafttreten des Arbeitsförderungsgesetzes (AFG) vor 50 Jahren in Deutschland zu feiern. Im Gegensatz $\mathrm{zu}$ früheren Jubiläen blieben diesmal jedoch Evaluierungen und Würdigungen der Bundesagentur für Arbeit oder des Instituts für Arbeitsmarkt- und Berufsforschung aus. Der vorliegende Beitrag sieht dies als Versäumnis und nimmt die hohen Standards der Arbeitsförderung, die durch das AFG gesetzt wurden, zum Ausgangspunkt, um zu fragen, inwiefern in der Arbeitsmarktpolitik der Bundesrepublik Deutschland seit 1969 sozialer Fortschritt erreicht wurde.

Dabei wird sozialer Fortschritt als ein innovatives Konzept verstanden, das den Erfolg sozialpolitscher Intervention nicht ausschließlich anhand ökonomischer Indikatoren misst. Vielmehr werden verschiedene normative Ziele, wie die Gewährleistung sozialer Rechte, Gleichheit bzw. Schutz vor Armut, miteinbezogen. Die Operationalisierung der einzelnen Indikatoren wird aus den aktuellen Strategie- und Positionspapieren verschiedener internationaler AkteurInnen wie der Weltbank, der Organisation for Economic Co-operation and Development (OECD) und der International Labour Organization (ILO) für eine zukunftsweisende Arbeitsmarkt- und Sozialpolitik abgeleitet. Für die entsprechende Analyse wird ein breites Verständnis von Arbeitsmarktpolitik zugrunde gelegt, d. h. es werden nicht nur das AFG und seine Nachfolgeregelungen im Sozialgesetzbuch (SGB) III, sondern auch die Reformen der verschiedenen Transferleistungssysteme, einschließlich der Reformen der Sozialhilfe und des SGB II, analysiert. Zudem wird punktuell auf relevante Leistungen in flankierenden Politikfeldern rekurriert.

Im Anschluss an die Diskussion des Forschungsstandes (1) wird sozialer Fortschritt in der Arbeitsmarktpolitik als Referenzkategorie anhand verschiedener Dimensionen operationalisiert (2). Diese werden genutzt, um verschiedene historische Entwicklungsstadien der Arbeitsmarktpolitik in Deutschland zu bewerten. Ausgangspunkt ist dabei das AFG selbst sowie damit einhergehende arbeitsmarktpolitische Entwicklungen in den ersten Jahren nach seinem Inkrafttreten (3). Im Anschluss werden die zahlreichen Reformgesetze des AFG kumulativ betrachtet. Dabei unterscheiden wir die Phase bis Ende der 1980er-Jahre, in der Kürzungen im Zuge der Haushaltskonsolidierung dominierten (4). Mit der Wiedervereinigung kam es zur erneuten Ausweitung von Finanzierung und MaßnahmenteilnehmerInnen, die jedoch vielfach mit weiteren Einschränkungen für individuelle
LeistungsempfängerInnen verknüpft wurden (5). Die Gesetze zur Modernisierung der Dienstleistungen am Arbeitsmarkt, die bis Mitte der 20ooer-Jahre verabschiedet wurden, leiten umfassende Reformen ein und werden als Paradigmenwechsel hin zur aktivierenden oder sozial investiven Arbeitsmarktpolitik verstanden. Gleichwohl erfolgt faktisch kein erneuter Ausbau aktiver Leistungen wie der Weiterbildung (6). Erst mit dem 2016 eingeführten Gesetz zur Stärkung der beruflichen Weiterbildung und des Versicherungsschutzes in der Arbeitslosenversicherung (AWStG) sowie dem Qualifizierungschancengesetz von 2018 (Bäcker 2018) deutet sich eine vorsichtige Trendwende an (7). Inwiefern damit die Standards des AFG in der Arbeitsförderung eingeholt bzw. überholt werden, wird im Fazit diskutiert (8).

\section{Forschungsstand}

Das AFG manifestierte den Wandel von einer eher markt-kompensatorischen und auf (passive) soziale Absicherung der ArbeitsmarktklientInnen abzielende Politik zugunsten der aktiven Förderung des Ausgleichs struktureller Defizite am Arbeitsmarkt (Altmann 2004), insbesondere über Qualifizierungs-, zum Teil aber auch über Arbeitsbeschaffungsmaßnahmen. Die Ende der 1990er-Jahre eingeleitete aktivierende Arbeitsmarktpolitik setzte dies fort, allerdings mit veränderter Zielsetzung. Diese beinhaltete einerseits die Förderung individueller Befähigungsfähigkeit und die Verschärfung der Arbeitspflicht sowie andererseits eine Universalisierung der Arbeitsmarktteilhabe (Dingeldey 2003: 97).

Indem das AFG also mit der passiven Arbeitsmarktpolitik verzahnt ist und viele Maßnahmen in der aktivierenden Arbeitsmarktpolitik fortgesetzt werden, umfasst der Forschungsstand nahezu die gesamte Arbeitsmarktpolitikforschung der Bundesrepublik. Da dies hier nicht darzustellen ist, wird im Folgenden auf explizite Evaluationen zum AFG bzw. nachfolgenden Gesetzen sowie auf exemplarisch ausgewählte Publikationen zum Übergang hin zur aktivierenden Arbeitsmarktpolitik rekurriert.

Mit dem AFG wurde der damaligen Bundesanstalt für Arbeit auch die Aufgabe der wissenschaftlichen Arbeitsmarktforschung übertragen und vor allem die Reformen seit Mitte der 20ooer-Jahre sind explizit mit einer Evaluationsaufforderung versehen. Entsprechend liegen zahlreiche Evaluationen einzelner arbeitsmarktpolitischer Instrumente vor (zusammenfassend siehe 
Bernhard et al. 2008; Caliendo/Steiner 2005; Heyer 2011; Heyer et al. 2012; Schmid 1987; Steiner/Hagen 2001). Die genannten Studien orientieren sich vielfach an Zielgrößen hinsichtlich des Übergangs bzw. der Dauer des Verbleibs in Beschäftigung (Sesselmeier 2017) - also an primär ökonomisch-quantifizierbaren Kriterien. Darüber hinaus liegen turnusmäßig verfasste Jubiläumswürdigungen vor (Bogedan 2009; Lampert 1989; Sell 1998). Analysen zum Wandel der Arbeitsmarktpolitik in historischer Perspektive nutzen das AFG als Projektionsfolie für nachfolgende Reformen, wobei die Integration des AFG ins SGB III im Jahr 1998 (Dingeldey/Gottschall 2001; Heinelt 2003; Schmid/Wiebe 1999; Sell 2000; Weishaupt 2011) oder die Gesetze zur Modernisierung der Dienstleistungen am Arbeitsmarkt (2003-2005) (Bothfeld et al. 2012; Dingeldey 2006; Eichhorst et al. 2008; Heinelt 2003) besonders hervorstechen. Zudem wird die Rolle der Arbeitsmarktpolitik zur Bewältigung der Folgen der Wiedervereinigung hervorgehoben (Heinelt/Weck 1998; Knuth 2018).

Dabei wird vor allem die Reformwelle zwischen 1998 und 2005 als grundlegender Wandel der Gestaltung der Transfersysteme für Arbeitslose wie auch der aktiven hin zur aktivierenden Arbeitsmarktpolitik verstanden. Die entsprechenden Reformen bildeten vielfach die Grundlage für die Diskussion des Paradigmenwechsels vom fürsorgenden zum aktivierenden oder sozialinvestiven Wohlfahrtsstaat (Dingeldey 2006, 2011a). Vielfach wird untersucht, inwiefern dies einen Pfadbruch mit dem etablierten deutschen Sozialmodell beinhaltet (als Auswahl siehe Dingeldey 2006, 2011a; Klenk 2009; Oschmiansky et al. 2007). Entsprechende Analysen üben oftmals Kritik an der Ambivalenz der verschiedenen Reformen (Dingeldey 2007). So wird einerseits z. B. auf die Verschärfung der Arbeitspflicht bzw. die Sanktionen verwiesen, die für Arbeitslose im SGB-II-Bezug eingeführt wurden. Andererseits werden flankierende Reformen der Familienpolitik mit Blick auf die Förderung der Erwerbsteilhabe von Frauen positiv herausgestellt (Dingeldey 2011b).

\section{Operationalisierung der Fragestellung}

Die vorliegende Analyse schließt an diese Arbeiten an nimmt jedoch zudem auf die aktuelle Debatte Bezug, welche die gesellschaftliche Entwicklung bzw. den sozialen Fortschritt nicht nur anhand ökonomischer Indikatoren misst (Greve 2017), sondern auch soziale Indikatoren wie Happiness und Well-being berück- sichtigt (Frey 2018; Layard 2005). In den vergangenen Jahren wurden dazu in verschiedenen nationalen und internationalen Kontexten Indices entwickelt, z. B. der Better Life Index der OECD (2011ff.), bei dem die Lebensqualität $u$. a. anhand verschiedener Indikatoren zu Beschäftigung, Zivilengagement und Sicherheit gemessen wurde. Der Bericht der deutschen Bundesregierung zu Well-being nennt u. a. Indikatoren $\mathrm{zu}$ guter Arbeit oder gleichen Bildungsbedingungen für alle (Bundesregierung 2017). Der für ein globales Assessment konstruierte social progress index berücksichtigt Dimensionen wie Basic Human Needs, Foundations of Well-being and Opportunity (Social Progress Imperative 2018). Die eher allgemeinen Indikatoren lassen sich nur bedingt auf die Arbeitsmarktpolitik übertragen, sodass es für den hier intendierten Zweck einer Präzisierung bedarf. Die genannten Indices zeigen jedoch die zunehmende Relevanz der Debatte, sozialen Fortschritt nicht ausschließlich ökonomisch zu definieren. Zudem wird eine anschauliche Definition des normativen Zielpunktes von sozialem Fortschritt in Bezug auf die Gestaltung von Institutionen und Policys sowie deren Outcomes gegeben, die als Ausgangspunkt dienen kann, nämlich ,the capacity of a society to meet the basic human needs of its citizens, establish the building blocks that allow citizens and communities to enhance and sustain the quality of their lives, and create the conditions for all individuals to reach their full potential“" (Social Progress Imperative 2018: 4).

Dieser allgemeine normative Zielpunkt lässt sich anhand der jüngsten Strategie- bzw. Positionspapiere von drei maßgeblichen, mit ökonomischer Entwicklung und der Gestaltung von Arbeit befassten internationalen Organisationen spezifizieren bzw. als Konzept des sozialen Fortschritts in Bezug auf die Entwicklung der Arbeitsmarktpolitik operationalisieren. Die OECD bzw. ihre Vorgängerorganisation, die Organisation for European Economic Co-operation (OEEC), war bei ihrer Gründung 1947 primär der Förderung des wirtschaftlichen Wachstums, der Beschäftigung und der Steigerung des Lebensstandards in den entwickelten Ländern verpflichtet und formuliert auf der Basis wissenschaftlicher Analysen Politikempfehlungen für Regierungen (OECD 2020). Die Weltbank ist eine Sonderorganisation der vereinten Nationen (UN) und zielt seit den 1960er-Jahren auf die weltweite Armutsbekämpfung. Sie kann konkrete finanzielle und technische Hilfe gewähren, wobei Vergabekonditionen oftmals anhand eigener wissenschaftlicher Analysen bestimmt werden (BMZ 2020). In den 1990er-Jahren 
waren die von beiden Organisationen empfohlenen Strategien vom neo-liberalen Paradigma geprägt und sahen die Deregulierung von Arbeitsstandards in Kombination mit dem Abbau von Sozialleistungen als Voraussetzung für die Schaffung von Arbeitsplätzen (Anner/Caraway 2010). Seitdem hat ein grundlegender Positionswechsel stattgefunden. Die OECD vertritt im Rahmen ihrer Job Strategy 2018 nun explizit eine sozial interventionistische Position und zielt auf soziale Inklusion (McBride 2019: 5751). Entsprechend werden nicht nur die Förderung qualifizierter Beschäftigung und die Qualifizierung der Arbeitskräfte zur Anpassung an die sich verändernden Arbeitsmärkte empfohlen, sondern auch der präventive Schutz vor Arbeitsmarktrisiken. Dabei werden auch die Umverteilung durch Steuerund Transfersysteme, einschließlich der gleichen Verteilung von Gewinnen zwischen Arbeitgebenden und Arbeitnehmenden, als Ziele benannt (OECD 2018). Ähnlich setzt die Weltbank in ihrem jüngsten Flaggschiff-Report THE CHANGING NATURE OF WORK die Priorität des Regierungshandelns bei Investitionen in Humankapital, in Verbindung mit Arbeitsregulierungen und einem universell garantierten sozialen Mindestschutz für alle Menschen - unabhängig von ihrem Erwerbsstatus. Die Finanzierung entsprechender Maßnahmen soll über Veränderungen der Steuersysteme erfolgen (World Bank 2019). Damit ergeben sich starke Übereinstimmungen zu den Forderungen der ILO, die seit 1946 ebenfalls eine Sonderorganisation der UN ist, aber bereits nach dem Ersten Weltkrieg als tripartistische Organisation gegründet wurde mit dem Ziel, allgemeine Arbeitsstandards zu entwickeln und deren Umsetzung weltweit zu fördern (ILO 2020). Hier ist also eine prinzipielle Befürwortung von Regelungen zum Schutz der Arbeitskräfte zu konstatieren. Konkret fordert die ILO in ihrem jüngsten Positionspapier eine Steigerung der Investitionen in die Fähigkeiten der Menschen; in gute und nachhaltige Arbeit sowie die institutionelle Gestaltung der Arbeitsverhältnisse durch Festsetzung von Mindeststandards (ILO 2019). Forderungen im Sinne einer universellen Arbeitsgarantie - Recht auf Arbeit - sowie die Sicherung fundamentaler Arbeitnehmerrechte als Teil umfassender sozialer Bürgerreche sind weiterhin Alleinstellungsmerkmale der ILO.

Die kurz skizzierten Ziele der sehr verschiedenen Organisationen zeigen programmatische Überschneidungen dahingehend, dass insbesondere die Qualität von Arbeit sowie die Qualifizierung der Arbeitskräfte von allen angesprochen wird. Zudem werden soziale
Inklusion, die soziale Mindestsicherung bzw. die Vermeidung von Armut sowie Umverteilungsziele in der einen oder anderen Form von allen Organisationen genannt. Dies kann als eine Art übergreifender Konsens hinsichtlich der Ziele des sozialen Fortschritts in Verbindung mit der Entwicklung von Arbeit interpretiert werden. Diese Ankerpunkte lassen sich auf die Entwicklung von Arbeit bzw. Arbeitsmarktpolitik beziehen und anhand der von den einzelnen Organisationen benannten Elemente als Zieldimension konkretisieren. Die Förderung von Aus- und Weiterbildung für alle lässt sich durch den von der OECD genannten präventiven Aspekt ergänzen und erscheint über die von der ILO favorisierte Standardisierung in Form der Gewährung eines Rechtsanspruchs in höchster Form verwirklicht. Die in unterschiedlichen Arten benannten Ziele der Armutsvermeidung bzw. der Mindeststandardsicherung lassen sich so interpretieren, dass Transfer- und Erwerbseinkommen jenseits der Armutsgrenze liegen sollen. Inklusion, Umverteilung bzw. die Verringerung sozialer Ungleichheit können in Verbindung mit der Forderung nach guter Arbeit an einer hohen Erwerbsbeteiligung in gesicherten Arbeitsverhältnissen wie auch anhand von Maßnahmen zur expliziten Förderung benachteiligter Gruppen gemessen werden.

Entsprechend lassen sich vier Zieldimensionen zur Beurteilung der Arbeitsmarktpolitik im Sinne des sozialen Fortschritts konkretisieren:

1. Die Förderung von Aus- und Weiterbildungsmaßnahmen bei Arbeitslosigkeit, einschließlich präventiver Förderung von Beschäftigten durch entsprechende Maßnahmen;

2. Ein universeller Rechtsanspruch auf entsprechende Fördermaßnahmen bzw. auf die Vermittlung angemessener und dauerhafter Beschäftigung;

3. Transfer- und Erwerbseinkommen jenseits der Armutsgrenze für alle BürgerInnen;

4. Eine hohe Arbeitsmarktteilhabe auch von benachteiligten Gruppen sowie die Verringerung sozialer Ungleichheit quasi als Outcome dieser Maßnahmen.

Da im Rahmen der hier skizzierten Fragestellung ein vergleichsweiser langer Zeitraum zu betrachten ist, beschränken wir uns auf zentrale Faktoren, anhand derer das Erreichen der genannten Ziele im Zeitverlauf untersucht wird. Es wird auf die Förderung von Weiterbildungsmaßnahmen fokussiert, indem die $\mathrm{Zu}$ - und Abnahme der Teilnehmendenzahlen sowie die Repräsentanz ausgewählter, besonders benachteiligter Grup- 
pen exemplarisch betrachtet werden. Zudem werden Zugangsbedingungen zu Förderungen berücksichtigt, wobei ein allgemeiner Rechtsanspruch als besonders fortschrittlich gilt. Angemessene Beschäftigung wird anhand der allgemeinen Struktur der Beschäftigungsverhältnisse beurteilt, wobei vor allem Beschäftigung im sogenannten Normalarbeitsverhältnis (Vollzeit, unbefristet) positiv bewertet wird. Teilzeitbeschäftigung gilt im Vergleich mit Vollzeit dagegen eher als Risiko für die soziale Absicherung und - aufgrund der geschlechtsspezifisch ungleichen Verteilung - auch als Indiz sozialer Ungleichheit. Bei der Beurteilung von Transfer- und Erwerbseinkommen wird auf Bezugspunkte wie die Armutsrisikoquote (6o Prozent des mittleren Einkommens) bzw. die Niedriglohnschwelle (6o Prozent des mittleren Lohns) rekurriert. Die Arbeitsmarktteilhabe wird anhand der Entwicklung der Beschäftigung bzw. der Höhe der Arbeitslosigkeit beurteilt, wobei die Integration benachteiligter Gruppen exemplarisch anhand der Beschäftigungsintegration von Frauen untersucht wird. Soziale Ungleichheit wird in Bezug auf Höhe und Zugang zu Transferleistungen sowie die Form der Arbeitsmarktintegration, einschließlich der Lohndifferenzierung bzw. der Entwicklung eines Niedriglohnbereichs bewertet.

Die genannten Indikatoren für die verschiedenen Dimensionen werden im Zeitverlauf analysiert, um den sozialen Fortschritt zu bestimmen. Einschränkend gilt es dabei jedoch festzuhalten, dass die entsprechenden Entwicklungen nicht originäre Outcomes des AFG sind, sondern durch eine Vielzahl weiterer Faktoren beeinflusst werden. Arbeitslosigkeit bzw. Beschäftigungsniveau sind nicht allein auf die Arbeitsmarktpolitik zurückzuführen, sondern in hohem Maße konjunktur- bzw. kontextabhängig. Entsprechend sind auch die demografische Entwicklung und die Veränderung geschlechtsspezifischer Rollenbilder und Erwerbsmuster zentrale Einflussfaktoren.

Die unterschiedenen Phasen der Arbeitsmarktentwicklung orientieren sich einerseits an wirtschaftlichen Krisen und politischen Herausforderungen, berücksichtigen andererseits aber auch maßgebliche Neuorientierungen der Arbeitsmarktpolitik. Die jeweiligen Daten werden anhand von Sekundärquellen bzw. durch Literatur- und Dokumentenanalyse erhoben. Eine umfassende Dokumentation aller Arbeitsmarktgesetze und Reformen beinhaltet Steffen (2019). Als Quellen werden bevorzugt Studien genutzt, die Daten der Bundesagentur für Arbeit oder des Instituts für Arbeitsmarkt- und Berufsforschung nutzen. Zum Teil wird aber auch auf Zeitreihen zurückgegriffen, die auf Basis dieser Daten in Sekundärquellen erstellt wurden, so z. B. Bothfeld et al. (2012) oder Knuth (2018).

\section{Das AFG und die Arbeitsmarktentwicklung bis Mitte der 1970er-Jahre}

Das AFG wurde bei seinem Inkrafttreten 1969 vom damals amtierenden Arbeitsminister der ersten großen Koalition Hans Katzer (CDU) als „Grundlage einer zukunftsweisenden Arbeitsmarkt- und Beschäftigungspolitik“" charakterisiert (Lampert 1989). Die aktive Arbeitsmarktpolitik sollte den Strukturwandel auf dem Arbeitsmarkt unterstützen und stand in einem Komplementärverhältnis zum Stabilitäts- und Wachstumsgesetz von 1967, welches das Vollbeschäftigungsziel im Rahmen einer keynesianischen Wirtschaftspolitik verfolgte. Zudem ist die Flankierung durch die Bildungsreformen zu nennen. Das Berufsbildungsgesetz von 1969 schaffte erstmals bundesweit einheitliche Regelungen und Qualitätsstandards für die dualen Ausbildungsberufe. In sukzessiven Reformen wurden auch Aufstiegsfortbildungen zum Meister, Techniker oder Fachwirt neu reguliert und fungierten als Referenzrahmen für die vom AFG geförderten Weiterbildungen (Bosch 2012). Umgekehrt wurde die damalige Bundesanstalt für Arbeit (BA) als Instrument der beruflichen Bildungspolitik der Bundesregierung apostrophiert (Lampert 1989). Der dem AFG zugeschriebene innovative Charakter basierte auf der Einlösung verschiedener oben genannter Zieldimensionen.

Als Kernaufgabe galt die Förderung der Berufsbildung, der Fortbildung und der Umschulung mit dem Ziel, unterwertige Beschäftigung $\mathrm{zu}$ vermeiden und beruflichen Aufstieg zu ermöglichen (Bosch 2012). In Folge des AFG kam es zu einer massiven Ausweitung der geförderten beruflichen Bildungsmaßnahmen auf 189.00o Teilnehmende im Jahr 1975 (Bosch 2012), es stellte damit das vom Finanzierungsaufwand her gewichtigste Tätigkeitsgebiet der BA dar (Oschmiansky/Ebach 2012). Die Instrumente des AFG sollten nicht nur zum Abbau, sondern auch präventiv zur Vermeidung von Arbeitslosigkeit sowie von Fachkräftemangel eingesetzt werden. Ein Rechtsanspruch auf individuelle Förderung der Berufsbildung galt daher nicht nur für Arbeitslose, sondern auch für Beschäftigte und schloss zu Beginn sogar den Anspruch auf ein Hochschulstudium ein. Dieser Anspruch wurde durch die Einführung des Unterhaltsgeldes untermau- 
ert, das großzügig bemessen war und ökonomische Unabhängigkeit garantierte (Lampert 1989).

Hinsichtlich Höhe und Struktur der Transferleistungen für Arbeitslose erfolgten unmittelbar mit dem AFG keine maßgeblichen Veränderungen. Allerdings hatten Ausweitungen des ArbeitslosengeldesLeistungsanspruchs im Sinne der Bezugsdauer und der Leistungshöhe bereits im Vorfeld stattgefunden (Altmann 2004). Entsprechend waren Arbeitslose leistungsberechtigt, die der Arbeitsvermittlung zur Verfügung standen und die Anwartschaftszeit durch mindestens sechs Monate sozialversicherungspflichtige Beschäftigung innerhalb von drei Jahren erfüllt hatten. Die Höhe des Arbeitslosengeldes (ALG) betrug Anfang der 1970er-Jahre für Ledige 62,5 Prozent des vormaligen Nettoentgelts. Die unbefristet gewährte Arbeitslosenhilfe (ALH) für Langzeitarbeitslose bzw. zuvor nur kurzfristig Beschäftigte war ebenfalls als Lohnersatzleistung - zwar auf geringerem Niveau (52,5 Prozent) - geregelt, erforderte allerdings eine Bedürftigkeitsprüfung (Schmidt 1998). Damit war das Arbeitslosengeld für Gruppen mit mindestens durchschnittlichem Lohn tendenziell armutsvermeidend angelegt. Faktisch war jedoch immer der Haushaltskontext ausschlaggebend.

Bis zur Wirtschaftskrise 1973 konnte bei anhaltendem Wirtschaftswachstum und vor dem Hintergrund keynesianischer Makropolitik weitgehend Vollbeschäftigung bewahrt werden (Abb. 1). Einschränkend ist hier allerdings anzumerken, dass die Erwerbsbeteiligung in Familienhaushalten noch weitgehend dem männlichen Ernährermodell folgte und damit ein deutlich geringerer Anteil der Bevölkerung erwerbstätig war (Dingeldey/Gottschall 2001). Gleichwohl war es ein erklärtes Ziel des AFG, ArbeitnehmerInnen mit besonderen Eingliederungserschwernissen wie Behinderte, ältere ArbeitnehmerInnen und Frauen explizit zu fördern (Lampert 1989). Obgleich Frauen nun - allerdings primär via Teilzeitbeschäftigung - in stärkerem Maße in den Arbeitsmarkt integriert wurden, blieb die Frauenerwerbsquote unter 50 Prozent (Schmid 1987).

Dies ging einher mit Regelungen im Transfersystem, die sich genderspezifisch auswirkten, indem insbesondere Frauen aufgrund von Familienphasen die genannten Rahmenfristen für den ALG-Bezug nicht erfüllten oder durch die Bedürftigkeitsprüfung aufgrund des Einkommens vom Ehepartner keine ALH erhielten. Vor allem alleinerziehende Mütter waren damit primär auf die 1961 eingeführten Leistungen des Bundessozialhilfegesetzes verwiesen, das auf Basis einer deutlich schärferen Bedürftigkeitsprüfung als die
ALH mittels sehr knapp kalkulierter Regelsätze ausschließlich auf Bedarfsdeckung zielte (Schmidt 1998). Zudem lagen die Leistungen überwiegend unterhalb der Armutsgrenze (Boss 2008). Zunächst waren jedoch weniger Arbeitslose als vielmehr ältere Menschen im Sozialhilfebezug dominant (Buhr et al. 1991).

Das AFG ist damit in Bezug auf die präventive Förderung von Weiterbildung sowie hinsichtlich des Rechtsanspruchs auf Fördermaßnahmen als besonders fortschrittlich zu betrachten. Die geschlechtsspezifische Förderung und Arbeitsmarktintegration ist für die damalige Zeit als innovativ zu sehen, obgleich die erreichte Erwerbsteilhabe von Frauen bis Mitte der 1970er-Jahre sehr gering blieb. Dies bestätigt, dass die Steigerung der Erwerbsteilhabe von Frauen und Müttern nicht allein durch die Arbeitsmarktpolitik beeinflusst wird, sondern in hohem Maße vom sozio-kulturellen Wandel sowie weiteren flankierenden Maßnahmen abhängig ist. Die differenten Leistungen von ALG und ALH einerseits und Sozialhilfe andererseits schrieben massive soziale Ungleichheiten zwischen dem Arbeitslosensicherungssystem und der Armenfürsorge weiter fort. Entsprechend sind in Bezug auf die Bekämpfung sozialer Ungleichheit allein innerhalb der Transfersysteme noch deutliche Defizite zu konstatieren.

\section{Rückschritte beim Rechtsanspruch und dem Präventionsgedanken im Zuge der Wirtschaftskrise in den 1970er- und 1980er-Jahren}

Infolge der Ölkrise 1973 änderten sich die wirtschaftlichen Parameter. Die Arbeitslosigkeit erhöhte sich auf zunächst knapp fünf Prozent, um infolge des zweiten Ölpreisschocks auf 9,3 Prozent im Jahr 1985 hochzuschnellen (siehe Abb. 1). Ein Kurswechsel zugunsten einer eher angebotsorientierten Wirtschaftspolitik in Kombination mit einer restriktiven Haushaltspolitik fand noch unter der SPD-geführten Regierung statt und wurde schließlich durch die seit 1982 von Helmut Kohl geführte CDU-Regierung mit Nachdruck fortgesetzt (Ostheim/Schmidt 2007a). Im Bereich der Arbeitsmarktpolitik ging dies mit klaren Rückschritten in Bezug auf die individuelle Förderung einher, obgleich die Gesamtausgaben aufgrund der Zunahme der Zahl der Transferempfänger stiegen (siehe Abbildung A1). Nach 1975 bestimmten daher erneut die primär krisenbedingten Ausgaben für Arbeitslosen- und Kurzarbeitergeld prozyklisch den Haushalt der BA - was faktisch einen erneuten Rückfall in die Dominanz passiver Leistungen bedeutete (OECD 2001). 
Im Bereich der beruflichen Weiterbildung erfolgte eine schrittweise Engführung der Zugangsberechtigung, indem 1976 die Leistungen der aktiven Arbeitsmarktpolitik zunächst auf den Kreis der BeitragszahlerInnen und 1982 auf Arbeitslose bzw. auf von Arbeitslosigkeit Bedrohte beschränkt wurden. Aber selbst für diese Gruppen wurde der Rechtsanspruch auf berufliche Qualifizierung aufgegeben. Das Unterhaltsgeld wurde in Stufen gekürzt und für Aufstiegsqualifizierungen nur noch als Darlehen gewährt (Steffen 2019). Vor diesem Hintergrund sanken die geförderten Weiterbildungen Mitte der 1970er-Jahre auf 173.000, um Ende der 1980er-Jahre erneut auf über 300.000 anzusteigen (Bothfeld et al. 2012), da sie partiell auch als Substitut für eine mangelnde Arbeitskräftenachfrage eingesetzt wurden. Die Einschränkung des Präventionsgedankens und der Förderung des beruflichen Aufstiegs veränderten die Struktur der Teilnehmenden: Während 1973 nicht einmal sechs Prozent der neu eingetretenen Teilnehmenden bei Maßnahmen der beruflichen Bildung zu den Arbeitslosen zählten, waren es Mitte der 1990er-Jahre etwa 95 Prozent (Oschmiansky/ Ebach 2012).

Wurden die Transferleistungen 1975 im Zuge der Armutsvermeidung während der zunächst als temporär wahrgenommenen Krise nochmals angehoben, wurden sie seit 1983 massiv gekürzt (Steffen 2019). Eine Einschränkung der Anspruchsberechtigten ergab sich insbesondere im Bereich der originären Arbeitslosenhilfe. Aufgrund der Zunahme der Langzeitarbeitslosigkeit stieg gleichwohl die Anzahl der Leistungsberechtigten. Entsprechend nahmen Zahl und Anteil der Alleinerziehenden und Arbeitslosen im Sozialhilfebezug in Folge der Wirtschaftskrisen insbesondere in den 1980er-Jahren massiv zu (Buhr et al. 1991). Der Anteil der Haushalte, die aufgrund von Arbeitslosigkeit in den Sozialhilfebezug fielen, stieg von 1981 11,5 Prozent auf 198933 Prozent aller Sozialhilfehaushalte. Umgekehrt machten die arbeitslosen SozialhilfeempfängerInnen 17 Prozent aller Arbeitslosen aus (Zimmermann 2001). Darüber hinaus gab es bereits das Phänomen der Working Poor, da knapp zehn Prozent der SozialhilfeempfängerInnen Erwerbseinkommen hatten (Buhr et al. 1991). Insgesamt nahm damit die armutsvermeidende Funktion der Arbeitslosen- und Sozialtransfers unter den Bedingungen der Wirtschaftskrise im Zeitverlauf massiv ab.

Das Ziel der Förderung der Arbeitsmarktteilhabe von Arbeitnehmenden mit besonderen Eingliederungserschwernissen wurde nicht nur eingeschränkt, sondern durch selektive Ausgliederung spezifischer Gruppen ersetzt, z. B. über die Rückführung von ausländischen ArbeitnehmerInnen und umfassende Frühverrentungsprogramme. Einführung und Ausdehnung des Erziehungsgeldes bzw. -urlaubs Mitte der 1980erJahre waren nicht primär auf die Erweiterung der Vereinbarkeit von Beruf und Familie ausgerichtet, sondern dienten vor allem auch der Arbeitsmarktentlastung (Dingeldey/Gottschall 2001). Vor diesem Hintergrund stieg die Erwerbsteilhabe der Frauen bis Ende der 1980er-Jahre nur sehr langsam auf 54 Prozent (Schmid 1987). Die mit dem Beschäftigungsförderungsgesetz 1985 eingeleitete Deregulierung der Arbeitsverhältnisse signalisierte zudem eine Abkehr vom Prinzip der Förderung dauerhafter Beschäftigung, da einerseits der Kündigungsschutz eingeschränkt und andererseits befristete Beschäftigungsverhältnisse erleichtert wurden. Gleichwohl stieg auch der Anteil der befristeten Beschäftigung zunächst nur langsam an und blieb bis 1996 weitgehend stabil bei 3,9 Prozent (IAB 2019a).

Aufgrund der restriktiven ökonomischen und fiskalischen Bedingungen sind in dieser Phase letztlich in allen hier untersuchten Dimensionen Rückschritte anstelle von sozialem Fortschritt zu konstatieren.

\section{Ausweitung der Teilnehmerlnnenzahlen aktiver Maßnahmen im Zuge der Wiedervereinigung bis Anfang der 2000er-Jahre}

Mit der Wiedervereinigung wurde das AFG bzw. dessen bis dahin vollzogene Re-Regulierungen auf die neuen Bundesländer übertragen und als Puffer für den Strukturwandel bzw. den damit verbundenen Rückgang der Arbeitskräftenachfrage eingesetzt (Oschmiansky/ Ebach 2012). Dabei wurde gleichwohl deutlich, dass das Instrumentarium der aktiven Arbeitsmarktpolitik auf die Beseitigung des Mismatchs von Angebot und Nachfrage der Arbeitskräfte ausgerichtet ist, aber mit der Kompensation eines massiven Nachfragedefizits von Arbeit und der Garantie des Vollbeschäftigungsziels ohne entsprechende makroökonomische Flankierung überfordert ist. Die Arbeitslosigkeit stieg 1995 bundesweit auf ca. 11 Prozent mit regionalem Schwerpunkt in Ostdeutschland (Abbildung 1), obgleich die Zahl der Geförderten in aktiven Maßnahmen ${ }^{1}$ sukzessive ausgebaut wurde. In diesem Kontext erreichten

1 Bezeichnend ist hier u. a. auch die Ausweitung der Beschäftigung schaffenden Maßnahmen auf 466.0oo im Jahr 1992 (Bothfeld et al. 2012). 
Abbildung 1: Arbeitslosenquoten auf Basis abhängiger ziviler Beschäftigung in Prozent - wirtschaftliche Krisen und politische Reformen Arbeitslosenquoten auf Basis abhängiger ziviler Erwerbspersonen (in Prozent)
Deutuschland, Vest- und Ostdeutschland
1950 bzw. 1991 bis 2018

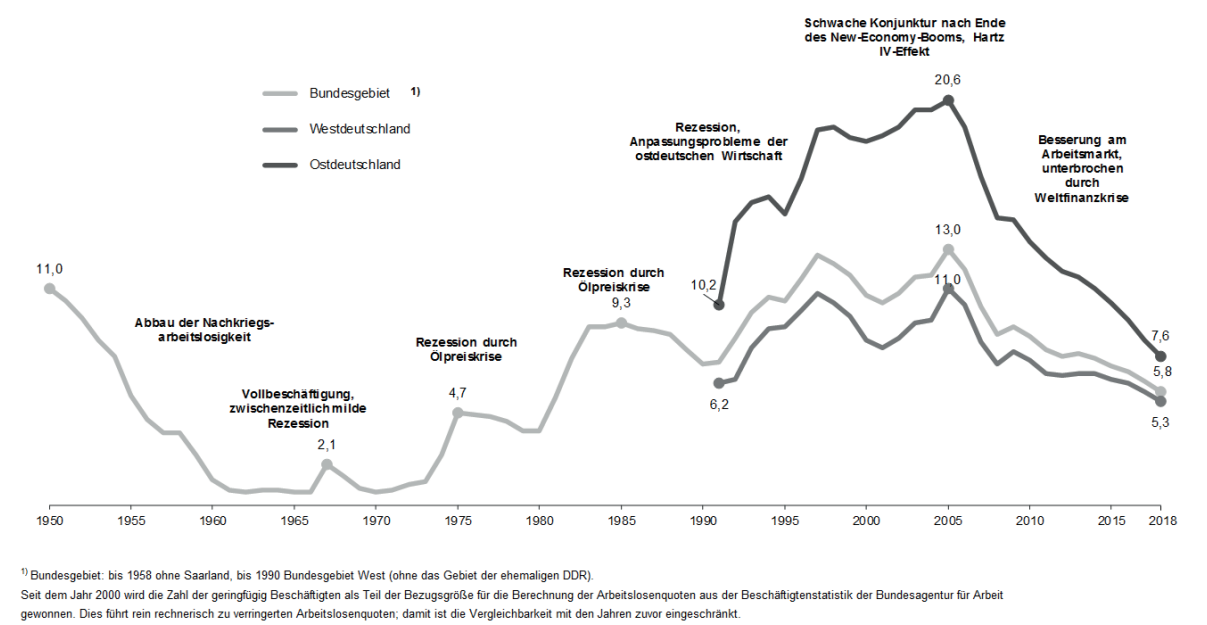

Quelle: Statistik der Bundesagentur für Arbeit Stand 11.01.2019; Online: www.statistik.arbeitsagentur.de/Navigation/Statistik/

Statistische-Analysen/Analyse-in-Grafiken/Jaehrliche-Zeitreihen/Jaehrliche-Zeitreihen-Nav [23.01.2019];

die Qualifizierungsmaßnahmen 1992 mit 863.000 TeilnehmerInnen (Bestand im Jahresdurchschnitt) einen absoluten Höchststand (Bothfeld et al. 2012). Gleichzeitig wurden jedoch die Kostenerstattung für Bildungsmaßnahmen ab 1989 und das Unterhaltsgeld ab 1994 nur noch als Kannleistung gewährt (Oschmiansky/Ebach 2012).

Mit der Arbeitslosigkeit stieg die Zahl der TransferleistungsempfängerInnen trotz der Einschränkung der Anspruchsberechtigung, sodass auch die Leistungshöhe gekürzt wurde. 1994 wurden das ALG (für Kinderlose) auf 6o Prozent und die ALH auf 53 Prozent des vorangegangenen Nettoentgeltes reduziert (Steffen 2019; siehe Abbildung A2) - womit das Ziel der Armutsprävention tendenziell aufgegeben und vor allem Langzeitarbeitslose zunehmend auf (ergänzende) Sozialhilfe verwiesen wurden: 1997 war in 41 Prozent der Haushalte im Sozialhilfebezug mindestens eine Person von Arbeitslosigkeit betroffen (Zimmermann 2001).

Nicht zuletzt die in Ostdeutschland bereits erfolgte Abkehr vom männlichen Ernährermodell trug dazu bei, dass auch im vereinigten Deutschland die Integration von Frauen in den Arbeitsmarkt wieder stärker gefördert wurde. Hier sind die Erweiterung von Rahmenfristen beim ALG aufgrund von Kindererziehungszeiten für Berufsrückkehrerinnen oder die Einführung einer Frauenförderquote bei der Maßnahmenförderung im Jahr 1992 zu nennen (Jaehrling 2012). Möglicherweise noch wichtiger für diese Entwicklung waren die Reformen von Erziehungsgeld und Erziehungsurlaub ab 2001, die nunmehr zunehmend auf eine Verstetigung der Erwerbsteilhabe von Frauen ausgerichtet wurden (Dingeldey 2006). Dies wurde flankiert durch die Ausweitung der Kinderbetreuung in Form des 1996 eingeführten Rechtsanspruchs auf einen Kindergartenplatz für Kinder ab drei Jahren.

Der erhöhte Anstieg der Erwerbsteilhabe von Frauen geht allerdings mit einer zunehmenden Segregation des Arbeitsmarktes und sich verschärfender sozialer Ungleichheit einher. Die Arbeitsmarktintegration von Müttern erfolgte vor allem in Westdeutschland überwiegend durch den Anstieg von Teilzeitarbeit, die 1998 knapp 14 Prozent der Gesamtbeschäftigung ausmachte (Statistisches Bundesamt 2001). Zudem arbeiteten 1999 3,6 Millionen Menschen in ausschließlich geringfügigen Beschäftigungsverhältnissen (Bothfeld et al. 2012: 371) - und damit in besonders prekären Beschäftigungsformen. Die befristete Beschäftigung stieg auf 5,1 Prozent im Jahr 1998 an (IAB 2019a).

Zur Bewältigung von Wirtschafts- und Strukturkrisen im Zuge der Wiedervereinigung wurden durch die insgesamt 115 Änderungsgesetze seit Inkrafttreten des AFG bis 1998 (Sell 1998) klare Rückschritte in Bezug auf den universellen Rechtsanspruch und die präventive Förderung durch Aus- und Weiterbildungsmaßnahmen realisiert. Ebenso wurde der Anspruch der Armutsvermeidung durch Transfers tendenziell aufgegeben. Die im Vergleich noch moderaten, aber gleichwohl positiven Entwicklungen bei der Erhöhung 
der Arbeitsmarktteilhabe von Frauen wurden durch die Maßnahmen der aktiven Arbeitsmarktpolitik lediglich moderiert und sind primär in der gesellschaftlichen Modernisierung und Individualisierung sowie im eher egalitären Familienerwerbsmodell Ostdeutschlands begründet (Jaehrling 2012). Insgesamt ist vor dem Hintergrund der durch Wirtschaftskrisen und Wiedervereinigung bedingten Herausforderungen bis zu diesem Zeitpunkt eine Rücknahme der im AFG formulierten progressiven Ziele der Arbeitsmarktpolitik in Deutschland zu konstatieren.

\section{Aktivierende bzw. sozial investive}

Arbeitsmarktpolitik und zunehmende soziale

Ungleichheit seit Ende der 1990er-Jahre bis 2009

Die Ideen eines aktivierenden bzw. sozialinvestiven Wohlfahrtsstaates fanden zunächst mit dem Arbeitsförderungs-Reformgesetz von 1998 Eingang in die deutsche Arbeitsmarktpolitik. Mit der Integration der Arbeitsförderung und der ALG-Leistungen ins SGB III wird das prioritäre Ziel der aktiven Arbeitsförderung zunächst auf den Ausgleich am Arbeitsmarkt sowie die Vermeidung von Arbeitslosigkeit reduziert und der Berufsschutz aufgegeben (AFRG 1997). Das JobAQTIV-Gesetz von 2001 nennt erstmals die Förderung individueller Beschäftigungsfähigkeit als Kernelement der Aktivierungsidee und reintegriert frühere Ziele wie die Förderung eines hohen Beschäftigungsstandes, die Verbesserung der Beschäftigungsstruktur, den Ausgleich zwischen Angebot und Nachfrage sowie die Vermeidung unterwertiger Beschäftigung ( $\$ 1$, SGB III nach JOB-AQTIV Gesetz 2001). Der Ansatz der aktivierenden Arbeitsmarktpolitik wird mit den Anfang der 20ooer-Jahre verabschiedeten Gesetzen für moderne Dienstleistungen am Arbeitsmarkt (bekannt als Hartz-I-IV-Reformen) bestätigt bzw. komplettiert. Entsprechend werden sie im Vergleich zum AFG als bahnbrechende Reform bezeichnet (Dingeldey 2011a; Ostheim/Schmidt 2007b).

Trotz der ambitionierten Ziele der aktivierenden oder sozialinvestiven Arbeitsmarktpolitik, die idealtypisch Prävention und die Förderung individueller Befähigung betont, werden in Deutschland die zur Bewältigung der mit der Wiedervereinigung verbundenen Probleme ausgedehnten Weiterbildungsmaßnahmen vor dem Hintergrund der nach 2005 erneut sinkenden Arbeitslosigkeit (siehe Abb. 2) abgebaut. Zudem steht der 2003 eingeführte Bildungsgutschein für die Neuausrichtung des Steuerungs- und Finanzie- rungsmodells des SGB III. Ein Rechtsanspruch auf Weiterbildung wird nicht eingeführt, sondern Maßnahmen der Arbeitsförderung bleiben Ermessensleistungen. Zudem wird die Anspruchsdauer auf Arbeitslosengeld in Verbindung mit einer Weiterbildung gekürzt. Die TeilnehmerInnenzahl bei den Maßnahmen der beruflichen Weiterbildung (Bestand) sinkt von knapp 380.000 im Jahr 2002 auf 142.000 im Jahr 2005 (Bundesagentur für Arbeit 2016: 60).

Erst zum Ende der Dekade erfolgt eine erneute Ausweitung der Zugangsbedingungen und die Stärkung des Präventionsgedankens, indem auch Beschäftigte ohne (aktuelle) Berufsausbildung gefördert werden können (Steffen 2019). 2009 wird ein erneuter Höchststand mit 264.000 TeilnehmerInnen in der beruflichen Weiterbildung erreicht, der dann allerdings 2018 wieder auf 166.000 sinkt $^{2}$ (Bundesagentur für Arbeit 2019).

Bei der Förderung von Frauen als benachteiligte Gruppe sind jedoch weiterhin Erfolge im Sinne des sozialen Fortschritts zu verzeichnen. Die Maßnahmen der Weiterbildung bzw. der Arbeitsförderung insgesamt sind - entsprechend des im Job-AQTIV-Gesetz explizit formulierten Ziels - weitgehend geschlechteregalitär verteilt. Dabei erscheint zentral, dass der Erfolg tatsächlich über das Konzept des AFG hinausgeht. Im Einklang mit den Zielen der Förderung von Beschäftigungsfähigkeit wurden arbeitsmarktpolitische Instrumente durch andere Politiken wie den Ausbau familienpolitischer Leistungen flankiert (Dingeldey 2011a). Zu nennen sind hier das einkommensabhängige Elterngeld seit 2007 und die Ausweitung des Rechtsanspruchs auf einen Betreuungsplatz für Kinder ab einem Jahr, der seit 2013 gilt, sowie der sukzessive Ausbau der Ganztagsschulen. Die Bündelung dieser Maßnahmen hat dazu beigetragen, dass die Erwerbsintegration der Frauen weiterhin massiv von 66,8 Prozent im Jahr 2005 auf 74 Prozent im Jahr 2017 anstieg. Für Männer betrug der entsprechende Anstieg der Erwerbsintegration lediglich zwei Prozentpunkte auf 82,4 Prozent 2017 (Bundesagentur für Arbeit 2019). Vor dem Hintergrund einer positiven ökonomischen Entwicklung stieg daher die Beschäftigung von 39,6 Millionen im Jahr 2005 quasi stetig an (Bundesagentur für Arbeit 2019), während die Arbeitslosigkeit sank (siehe Abbildung 1).

2 Zahlenreihen aufgrund verschiedener Quellen sowie aufgrund wechselnder Maßnahmen nicht durchgängig kongruent. 
Trotz des hohen Beschäftigungsstandes kann der Anspruch, Arbeitslose in angemessene und dauerhafte Beschäftigung $\mathrm{zu}$ vermitteln, nicht vorrangig verwirklicht werden. Beispielsweise wurden 20 Prozent der SGB-II-LeistungsbezieherInnen, die 2010 eine Erwerbstätigkeit aufnahmen, in die Zeitarbeitsbranche integriert (IAB 2019b). Insbesondere junge Menschen (16-24 Jahre) waren $2016 \mathrm{zu}$ knapp einem Viertel befristet beschäftigt, während dies auf nur etwa sieben Prozent bei den 35- bis 44-Jährigen zutraf (WSI 2016).

Der mit den Hartz-Reformen erfolgte Umbau der Transfersysteme für Arbeitslose 2005 ist jedoch tendenziell als Abbau von Leistungen zu interpretieren. Weitere Kürzungen des versicherungsbasierten Arbeitslosengeldes fanden Anfang der 20ooer-Jahre mit dem Gesetz zu Reformen am Arbeitsmarkt statt. Hartz IV zementiert dann die Dualisierung des Systems, indem einerseits das veränderte versicherungsbasierte Arbeitslosengeld (ALG I) nach SGB III beibehalten und andererseits die bedarfsgeprüfte Grundsicherung (ALG II) durch die Zusammenführung von Arbeitslosenhilfe- und Sozialhilfeleistungen für Erwerbsfähige geschaffen und nach SGB II geregelt wird (Dingeldey 2011b). ${ }^{3}$ Die in den Vorgängersystemen (insbesondere der Sozialhilfe) bereits angelegte Arbeitspflicht wurde im Zusammenhang mit dem Prinzip des Forderns im SGB II eher verstärkt, da jede Art von atypischen Beschäftigungen als zumutbar gilt (Knuth 2018; Steffen 2019).

Der steigende Anteil der ALG-II-BezieherInnen an den Arbeitslosen insgesamt hat den Bedeutungsverlust des Versicherungssystems (Rosenthal 2012) weiter zementiert (siehe Abbildung A1). Zudem stieg der Anteil der Erwerbstätigen (mit mehr als 15 Stunden pro Woche), die auf ergänzende Leistungen nach SGB II angewiesen sind, 2018 auf 14 Prozent (Bundesagentur für Arbeit 2019), darunter etwa 17 Prozent mit Vollzeitbeschäftigung (IAB 2018: 133). Da vor allem Leistungen der Mindestsicherung im Allgemeinen unter der Armutsschwelle liegen (Dingeldey 2015), ist damit die Armutsprävention weiter gesunken.

Ungleichheit am Arbeitsmarkt zeigt sich insbesondere über die Zunahme atypischer Beschäftigung. Der Anteil befristeter Beschäftigung insgesamt stieg von 6,4

3 Für ArbeitslosenhilfebezieherInnen in kleinen Haushalten, die auch als Langzeitarbeitslose statusorientierte Leistungen erhalten hatten und (nach einer Übergangsfrist) auf das Niveau der vorherigen Sozialhilfe zurückgeworfen wurden, beinhaltete dies massive Kürzungen ihrer Transfers.
Prozent im Jahr 2005 auf 8,3 Prozent im Jahr 2018 (IAB 2019a) und wird zudem durch einen Anstieg der Leiharbeit begleitet (knapp drei Prozent der Erwerbstätigen 2010 (IAB 2019b)). Insgesamt ist der Anteil der MiniJobs - als ausschließlich geringfügige Beschäftigungsform ohne Sozialleistungsansprüche - mit 10,6 Prozent aller Beschäftigten 2018 recht hoch (Bundesagentur für Arbeit 2019). Neben dem Rückgang der Tarifdeckung und dem strukturellen Wandel hin zu geringer entlohnten Dienstleistungen dürften entsprechende Entwicklungen maßgeblich dazu beigetragen haben, dass der Niedriglohnsektor in Deutschland bis 2014 auf über 20 Prozent der Gesamtbeschäftigung anwuchs (Dingeldey et al. 2017).

Der diskursive Paradigmenwechsel hin zur aktivierenden Arbeitsmarktpolitik wird in Deutschland zunächst weitgehend als Kürzung von Maßnahmen und Leistungen umgesetzt. Positiv ist jedoch, dass die weitere Integration von Frauen bzw. Müttern in Koordination mit der Familienpolitik gefördert wird. Im Zusammenspiel mit der Flexibilisierung der Arbeitsformen und dem Rückgang der Tarifbindung tragen jedoch alle diese Entwicklungen zu verringertem sozialen Schutz von ArbeitnehmerInnen, vor allem aber von Langzeitarbeitslosen oder nur kurzfristig Beschäftigten, sowie zu zunehmender sozialer Ungleichheit bei.

\section{Explizite Stärkung der Weiterbildung und Qualifizierung seit 2009}

Aktuell nimmt die Förderung von Jugendlichen mit erschwerten Vermittlungsperspektiven sowie benachteiligten Auszubildenden einen Schwerpunkt in der Arbeitsförderung ein (Knuth 2018). Mit dem Gesetz zur Neuausrichtung arbeitsmarktpolitischer Instrumente 2009 wurde hier ein Rechtsanspruch auf berufsvorbereitende Bildungsmaßnahmen zum nachträglichen Erwerb des Hauptschulabschlusses bzw. eines vergleichbaren Abschlusses eingeführt. Zudem wurde die Berufsbildungsbeihilfe während der ersten Ausbildung eingeführt (Steffen 2019). 2018 wurden durch entsprechende Maßnahmen (inklusive Berufseinstiegsbegleitung, assistierte Ausbildung, ausbildungsbegleitende Hilfen etc.) über 190.000 Jugendliche gefördert (Bundesagentur für Arbeit 2019; siehe Abbildung A2). Zudem erfolgte mit dem Qualifizierungschancengesetz 2019 eine Ausweitung des Anspruchs auf Qualifizierungsmaßnahmen auf alle ArbeitnehmerInnen - sodass auch Beschäftigte erneut profitieren können. Zuschüsse sowohl zu den Lehrgangs- als 
auch Lohnkosten sind allerdings in Abhängigkeit der Betriebsgröße gestaffelt (Steffen 2019). Entsprechend findet eine erneute Zunahme von Weiterbildungsmaßnahmen statt.

Der amtierende Arbeitsminister Hubertus Heil hat des Weiteren das Arbeit-von-morgen-Gesetz auf den Weg gebracht (liegt Mitte März 2020 als Kabinettsbeschluss vor), das zusätzliche Weiterbildungsförderungen und Maßnahmen gegen den Konjunkturabschwung ankündigt. Unter anderem sollen dabei Geringqualifizierte einen Rechtsanspruch erhalten, mit Förderung einen Berufsabschluss nachzuholen. Anknüpfend an das Qualifizierungschancengesetz soll zudem Kurzarbeit in Konjunkturkrisen ausgeweitet und mit Qualifizierung verbunden werden, indem dies stärker durch Zuschüsse der BA gefördert wird (Handelsblatt 2019). Die Umsetzung dieser Regelungen nähert sich damit dem im AFG ehemals formulierten Rechtsanspruch auf Weiterbildung an.

Die fortgesetzte Ausweitung der Erwerbsteilhabe und vor allem auch die Reduzierung von geschlechtsspezifischen Ungleichheiten bei der Erwerbsteilhabe und der Arbeitsförderung sind als sozialer Fortschritt im Sinne der oben genannten Kriterien zu sehen. So ist nicht zuletzt aufgrund der aktuell positiven Konjunkturentwicklung ein Anstieg der Beschäftigung auf 44,8 Millionen im Jahr 2018 (Bundesagentur für Arbeit 2019) zu verzeichnen. Unterstützt durch die demografische Entwicklung (Knuth 2018) sank die Arbeitslosenquote auf 5,2 Prozent (Abbildung 1).

Ambivalenzen ergeben sich jedoch mit Blick auf das ebenfalls propagierte Ziel der Verringerung sozialer Ungleichheit: ALG-I-EmpfängerInnen profitieren deutlich mehr vom Aufschwung als ALG-II-BezieherInnen. Während sich die Zahl der ALG-I-BezieherInnen zwischen 2005 und 2018 von 1,42 auf 0,72 Millionen halbierte, nahmen ALG-II-BezieherInnen lediglich von 2,68 auf 1,52 Millionen ab (Bundesagentur für Arbeit 2019). Dies geht damit einher, dass das mit den Hartz-Reformen verfolgte Ziel, frühere SozialhilfeempfängerInnen in die Maßnahmen der aktiven Arbeitsmarktpolitik gleichberechtigt einzubeziehen, nicht erreicht wurde. De facto sind ALG-II-BezieherInnen 2018 mit einem Drittel der Teilnehmenden bei Maßnahmen der beruflichen Weiterbildung stark unterrepräsentiert (Bundesagentur für Arbeit 2019).

Gleichzeitig sind Segregation und Segmentation des Arbeitsmarktes aufgrund der fortgesetzten Zunahme bzw. des hohen Bestands atypischer Beschäftigungsformen extrem stark ausgeprägt: Während Männer konstant zu über 60 Prozent im Normalarbeitsverhältnis beschäftigt sind, gilt dies nur noch für knapp 30 Prozent der Frauen (Dingeldey 2015). Diese sind mit über drei Millionen aber fast doppelt so häufig in Mini-Jobs beschäftigt wie Männer (Bundesagentur für Arbeit 2016). Entsprechend ist auch ihr Anteil am Niedriglohnsektor deutlich ausgeprägter. 2017 waren laut Statistischem Bundesamt knapp acht Prozent der Erwerbstätigen und 57,2 Prozent der Erwerbslosen armutsgefährdet (Statista 2019).

Aktuell ist der Rechtsanspruch auf Aus- und Weiterbildung zwar noch nicht universell, aber es hat eine sehr starke Annäherung an den Standard des AFG stattgefunden. Dies gilt auch für die präventive Teilnahme an Weiterbildung. Zudem zielen Maßnahmen wie assistierte Ausbildung oder ausbildungsbegleitende Hilfen insbesondere auf die Integration von Jugendlichen in gute Arbeit, was über die Instrumente des AFG hinausgeht und - vor dem Hintergrund einer veränderten Problemlage - klar als sozialer Fortschritt interpretiert werden kann. Dies gilt auch für die weiterhin gestiegene Frauenerwerbsbeteiligung.

Trotz einzelner Maßnahmen wie der Einführung des Mindestlohns und Equal-Pay-Gesetzen, wie beispielsweise in Bezug auf die Leiharbeit, konnte der gestiegenen sozialen Ungleichheit am Arbeitsmarkt und der Armut allerdings noch kaum Einhalt geboten werden, sodass in Bezug auf diesen Aspekt ein klarer Rückschritt im Vergleich zum Einführungszeitpunkt des AFG zu konstatieren ist. Die Einführung des Mindestlohns ist in diesem Zusammenhang positiv zu sehen, hat aber eine durchaus begrenzte Wirkung (Mindestlohnkommission 2018). Zudem wären Maßnahmen der sozialen Sicherung erforderlich, die stärker auf die Diskontinuität von Erwerbsverläufen eingehen, wobei die Einführung der Grundrente ebenfalls als ein erster Schritt in diese Richtung zu sehen ist.

\section{Fazit}

Die Beurteilung des AFG als besonders innovativ und fortschrittlich - zu Zeiten seiner Einführung - gilt auch noch, wenn wir die aktuellen Ziele und Normen internationaler Organisationen für die zukünftige Entwicklung der Arbeitsmarktpolitik zugrunde legen. Entsprechend ist die Frage, ob in den vier untersuchten Dimensionen in den vergangenen 50 Jahren ein sozialer Fortschritt der deutschen Arbeitsmarktpolitik hinsichtlich der Entwicklung arbeitsmarktpolitischer Zielsetzungen und deren Umsetzung erreicht wurde, überwiegend negativ zu beantworten: 
1. Die (präventive) Förderung von Aus- und Weiterbildung zeigt ein Auf und Ab, das erneut auf Expansion gerichtet ist: Nach den massiven Rückschritten seit Mitte der 1970er-Jahre, der durch die Wiedervereinigung bedingten Expansion der Maßnahmen zur Kompensation des Beschäftigungseinbruchs in den neuen Bundesländern und deren erneutem Abbau Anfang der 200oer-Jahre erfolgte 2019 eine erneute Ausweitung der Anspruchsberechtigung. Obgleich die Zahlen der im Rahmen von Aus- und Weiterbildungsmaßnahmen geförderten Personen 1975 und 2018 nahezu gleich hoch waren, signalisiert dies angesichts der (auch durch die Wiedervereinigung) deutlich gestiegenen Zahl der Erwerbstätigen und einer ähnlich hohen Arbeitslosenquote einen relativen Bedeutungsverlust der aktiven Förderung. Die erneute Erweiterung von Zugangskriterien wie auch die Ausdehnung des Anspruchs auf alle Beschäftigten lässt eine erneute Steigerung der Inanspruchnahme auch durch Beschäftigte erwarten und damit eine Wiederherstellung der präventiven Förderung.

2. Beim universellen Rechtsanspruch auf Fördermaßnahmen und der Vermittlung in angemessene und dauerhafte Beschäftigung bestehen klare Rückschritte gegenüber dem AFG: Lediglich Jugendlichen wird ein Rechtsanspruch auf das Nachholen eines Hauptschulabschlusses gewährt - das Recht auf Aus- und Weiterbildung gilt es noch (wieder-)einzuführen. Zudem wurde das im AFG verankerte Prinzip des Berufsschutzes bei der Vermittlung der Arbeitslosen weitgehend aufgegeben. De facto erfolgt ein hoher Anteil der Übergänge von Arbeitslosigkeit in atypische Beschäftigungsformen, insbesondere Leiharbeit und befristete Beschäftigung.

3. Transfer- und Erwerbseinkommen jenseits der Armutsgrenze werden immer weniger erreicht: Indem die Niedriglohnbeschäftigung wie auch atypische Arbeitsverhältnisse zunehmen, steigt auch die Armut in Erwerbstätigkeit. Dies bedingt, dass immer häufiger nur geringe Anwartschaften beim ALG I erreicht und ergänzend Mindestsicherungsleistungen bezogen werden. Aufgrund der geringen Höhe des ALG II aber ist das Ziel armutssichernder Transfers für (Langzeit-)Arbeitslose immer weniger erreicht.

4. Damit ist allein die gestiegene Arbeitsmarktteilhabe klar als sozialer Fortschritt zu interpretieren, wobei die Erwerbsintegration von Frauen bzw. Müttern als benachteiligter Gruppe besonders positiv hervorzuheben ist. Hier wurde der allgemeine gesellschaftliche Wandel hin $\mathrm{zu}$ einer stärker geschlechteregalitären Arbeitsteilung durch die geschlechterproportionale Arbeitsförderung, insbesondere aber auch den flankierenden Ausbau der Kinderbetreuung und der Familienpolitik positiv beeinflusst. Ambivalenzen bestehen jedoch darin, dass keine generelle Verringerung sozialer Ungleichheit erreicht wurde, da die Ungleichheit in Beschäftigung aufgrund der Zunahme atypischer Beschäftigung und der Expansion des Niedriglohnsektors zugenommen hat, wovon Frauen überproportional stark betroffen sind.

Über das AFG bereits hinausgehend ist dagegen der Ansatz aktivierender Arbeitsmarktpolitik, Beschäftigungsfähigkeit durch Maßnahmen zu erreichen, welche die Arbeitsmarktpolitik flankieren. In der Bundesrepublik wurde dies - wie gezeigt - in Verbindung mit der Familienpolitik weitgehend umgesetzt, wenn auch hier der Bedarf an Kinderbetreuungsmöglichkeiten noch nicht voll gedeckt erscheint. Ebenfalls angelegt ist, dass beispielsweise Jugendliche ohne bzw. mit geringen Schulabschlüssen und Langzeitarbeitslose durch eine Ausweitung unterstützender sozialpädagogischer und gesundheitsfördernder Maßnahmen oder Schuldenberatung gefördert werden. Hier gibt es allerdings bislang viel Kritik, dass entsprechende Angebote in der Praxis nicht ausreichend angeboten werden.

Indem die aktuellen Herausforderungen einerseits durch Fachkräftemangel und andererseits durch die erneute Freisetzung von Arbeitskräften aufgrund der Prozesse der weiteren Digitalisierung und Globalisierung gekennzeichnet sind, haben sie durchaus Ähnlichkeit mit der Situation von 1969, die durch Strukturwandel geprägt war. Es überrascht also nicht, dass jüngst eine Wiederannäherung an die Ziele des AFG bereits stattgefunden hat. Die Fortsetzung könnte helfen, neue Herausforderungen zu bewältigen:

Der Rechtsanspruch auf Aus- und Weiterbildung für Beschäftigte könnte u. a. dazu beitragen, dass MigrantInnen mit mangelnden Sprachkenntnissen als neuere benachteiligte Gruppen quasi stufenweise, zunächst über niederschwellige Beschäftigung, in den Arbeitsmarkt integriert, dann aber für hochwertige Beschäftigungen qualifiziert werden könnten - ohne erneut arbeitslos werden zu müssen, um gefördert zu werden.

Um also in der gegenwärtigen Situation sozialen Fortschritt in der Arbeitsmarktpolitik entlang der von internationalen Organisationen gestützten Kriterien zu erreichen, scheint in Deutschland die partielle Re-Orientierung an den mehr als 50 Jahre alten Zielen und Leitlinien des AFG in Kombination mit neueren Konzepten zur Förderung der Beschäftigungsfähigkeit adäquat. 


\section{Literatur}

AFRG (1997): Arbeitsförderungsreformgesetz. Bundesgesetzblatt 1997 (20).

Altmann, G. (2004): Aktive Arbeitsmarktpolitik. Entstehung und Wirkung eines Reformkonzepts in der Bundesrepublik Deutschland. Stuttgart: Steiner.

Anner, M./Caraway, T. (2010): International Institutions and Workers' Rights: Between Labor Standards and Market Flexbility. Studies in Comparative International Development, 45, 151-169.

Bernhard, S./Hohmeyer, K./Jozwiak, E./Koch, S./Kruppe, T. (2008): Aktive Arbeitsmarktpolitik in Deutschland und ihre Wirkungen. IAB-Forschungsbericht 2008,2. Nürnberg: IAB.

BMZ (2020): Weltbankgruppe. Online: https://www.bmz. $\mathrm{de} / \mathrm{de} / \mathrm{ministerium/wege/multilaterale \_ ez/akteure/welt-}$ bank/index.html [28.02.2020].

Bogedan, C. (2009): 40 Jahre aktive Arbeitsmarktpolitik in Deutschland - Beitrag zu einer Bilanz. WISO direkt. Analysen und Konzepte zur Wirtschafts- und Sozialpolitik. Bonn: FES.

Bosch, G. (2012): Berufliche Weiterbildung in Deutschland 1969 bis 2010: Entwicklung und Reformoptionen. In: Bothfeld, S./Sesselmeier, W./Bogedan, C. (Hg.): Arbeitsmarktpolitik in der sozialen Marktwirtschaft. Vom Arbeitsförderungsgesetz zum Sozialgesetzbuch II und III. 2. überarbeitete Aufl. Wiesbaden: Springer VS, 106-125.

Boss, A. (2008): Zur Entwicklung des Anspruchslohns in Deutschland. Kiel Working Paper, 1463.

Bothfeld, S./Sesselmeier, W./ Bogedan, C. (2012): Arbeitsmarktpolitik in der sozialen Marktwirtschaft. Vom Arbeitsförderungsgesetz zum Sozialgesetzbuch II und III. 2. überarbeitete Aufl. Wiesbaden: Springer VS.

Buhr, P./Leisering, L./Ludwig, M./Zwick, M. (1991): Armutspolitik und Sozialhilfe in vier Jahrzehnten. In: Wollmann, H. (Hg.): Die alte Bundesrepublik. Kontinuität und Wandel. Opladen: Springer, 502-546.

Bundesregierung (2017): Bericht der Bundesregierung zur Lebensqualität in Deutschland. Online: https://www.gutleben-in-deutschland.de/SiteGlobals [03.03.2020].

Bundesagentur für Arbeit (2016): Der Arbeitsmarkt in Deutschland. Zeitreihen bis 2015. Analytikreport der Statistik. Nürnberg: Bundesagentur für Arbeit.

- (2019): Der Arbeitsmarkt in Deutschland 2018. Berichte: Blickpunkt Arbeitsmarkt. Amtliche Nachrichten der Bundesagentur für Arbeit, 66 (2)

Caliendo, M./Steiner, V. (2005): Aktive Arbeitsmarktpolitik in Deutschland: Bestandsaufnahme und Bewertung der mikroökonomischen Evaluationsergebnisse. Zeitschrift für ArbeitsmarktForschung, 38 (2/3), 396-418.

Dingeldey, I. (1998): Geringfügige Beschäftigung und soziale Absicherung. Soziale Sicherheit, 47 (12), 411-420.
- (2003): Politikfeldübergreifende Koordination als neue Steuerungsform im aktivierenden Sozialstaat. Österreichische Zeitschrift für Politikwissenschaft, 32 (1), 97-107.

- (2006): Aktivierender Wohlfahrtsstaat und sozialpolitische Steuerung. Aus Politik und Zeitgeschichte, 8-9, 3-9.

- (2007): Wohlfahrtsstaatlicher Wandel zwischen „Arbeitszwang" und „Befähigung“. Eine vergleichende Analyse aktivierender Arbeitsmarktpolitik in Deutschland, Dänemark und Großbritannien. Berliner Journal für Soziologie, 17 (2), 189-209.

- (2011a): Der aktivierende Wohlfahrtsstaat. Governance der Arbeitsmarktpolitik in Dänemark, Großbritannien und Deutschland. Frankfurt: Campus.

- (2011b): Germany: Moving towards Integration whilst Maintaining Segmentation. In: Clasen, J./Clegg, D. (Hg.): Unemployment Protection Systems and Labour Market Change in Europe. Oxford: Oxford University Press, 55-74.

- (2015): Bilanz und Perspektiven des aktivierenden Sozialstaats. Aus Politik und Zeitgeschichte, 10, 33-40.

Dingeldey, I./Etling, A./Kathmann, T./de Beer, P. (2017): Niedriglohnentwicklung und Lohnungleichheit im Vergleich - Die Rolle kollektiver Akteure. WSI Mitteilungen, 70 (7), 499-507.

Dingeldey, I./Gottschall, K. (2001): Alte Leitbilder und neue Herausforderungen: Arbeitsmarktpolitik im konservativ-korporatistischen Wohlfahrtsstaat. Aus Politik und Zeitgeschichte, 21, 31-38.

Eichhorst, W./Grienberger-Zingerle, M./Konle-Seidl, R. (2008): Activation Policies in Germany: From Status Protection to Basic Income Support. In: Eichhorst, W./ Kaufmann, O./Konle-Seidl, R. (Hg.): Bringing the Jobless into Work? Experiences with Activation Schemes in Europe and the US. Heidelberg: Springer, 17-68.

Frey, B. S. (2018): Economics of Happiness. Cham: Springer.

Greve, B. (2017): How to Measure Social Progress? Social Policy \& Administration, 51 (7), 1002-1022.

Handelsblatt (27.01.2020): Union und SPD tun sich mit Einigung beim „Arbeit von Morgen“-Gesetz schwer.

Heinelt, H. (2003): Arbeitsmarktpolitik - von „versorgenden“ wohlfahrtsstaatlichen Interventionen zur ,aktivierenden" Beschäftigungsförderung. In: Gohr, A./SeeleibKaiser, M. (Hg.): Sozial- und Wirtschaftspolitik unter Rot-Grün. Wiesbaden: Westdeutscher Verlag, 125-146.

Heinelt, H./Weck, M. (1998): Arbeitsmarktpolitik. Vom Vereinigungskonsens zur Standortdebatte. Opladen: Leske + Budrich.

Heyer, G./Koch, S./Stephan, G./Wolff, J. (2011): Evaluation der aktiven Arbeitsmarktpolitik. Ein Sachstandsbericht für die Instrumentenreform 2011. IAB Discussion paper, 17/2011, Nürnberg: IAB.

Heyer, G./Koch, S./Stephan, G./Wolff, J. (2012): Evaluation der aktiven Arbeitsmarktpolitik: Ein Sachstandsbericht für die Instrumentenreform 2011. Journal for Labour Market Research, 45 (1), 41-62. 
IAB (2019a): Befristete Beschäftigung in Deutschland. Stand 2019. Online: https://www.iab.de/de/daten/arbeitsmarktentwicklung.aspx [11.07.2019].

- (2019b): Zeitarbeit. Übergänge in die und aus der Arbeitslosenversicherung. Stand 29.07.2015. Online: https:// www.iab.de/de/daten/arbeitsmarktentwicklung.aspx [11.07.2019].

ILO (2019): Work for a brigher Future. Global Commission on the Future of Work. Geneva: International Labour Organization.

- (2020): History of the ILO. Online: https://www.ilo.org/ global/lang--en/index.htm [28.02.2020].

Jaehrling, K. (2012): Gleichstellung und Aktivierung -Wahlverwandtschaft oder Stiefschwestern? In: Bothfeld, S./ Sesselmeier, W./Bogedan, C. (Hg.): Arbeitsmarktpolitik in der sozialen Marktwirtschaft. Vom Arbeitsförderungsgesetz zum Sozialgesetzbuch II und III. 2. überarbeitete Aufl. Wiesbaden: Springer VS, 177-190.

Deutscher Bundestag (2001): Gesetz zur Reform der arbeitsmarktpolitischen Instrumente (Job-AQTIV-Gesetz). Bundesgesetzblatt Jahrgang 2001 Teil I Nr. 66.

Klenk, T. (2009): Vom Arbeitsförderungsgesetz zum Sozialgesetzbuch II und III: Pfadwechsel in der korporatistischen Arbeitsverwaltung? In: Bothfeld, S./Sesselmeier, W./Bogedan, C. (Hg.): Arbeitsmarktpolitik in der sozialen Marktwirtschaft. Vom Arbeitsförderungsgesetz zum Sozialgesetzbuch II und III. 2. überarbeitete Aufl. Wiesbaden: Springer VS, 205-219.

Knuth, M. (2018): Arbeitsmarktpolitik als Inklusionsprojekt? WSI-Mitteilungen, 71 (6), 456-467.

Lampert, H. (1989): 20 Jahre Arbeitsförderungsgesetz. Mitteilungen aus der Arbeitsmarkt- und Berufsforschung, 22 (2), 173-186.

Layard, R. (2005): Happiness: Lessons from a new science. London: Penguin Books

Mindestlohnkommission (2018): Zweiter Bericht zu den Auswirkungen des gesetzlichen Mindestlohns. Bericht der Mindestlohnkommission an die Bundesregierung nach $\$ 9$ Abs. 4 Mindestlohngesetz, Berlin.

McBride, St./Watson, J. (2019): Reviewing the 2018 OECD Jobs Strategy - anything new under the sun? Transfer: European Review of Labour and Research, 25 (2), 149-163.

OECD (2001): Employment Outlook. Paris: OECD.

- (2011ff.): Better Life Index. Online: http://www.oecdbetterlifeindex.org/de/\#/111111111111 [26.02.2020].

- (2018): Good Jobs for All in a Changing World of Work. The OECD Jobs Strategy. Paris: OECD Publishing.

- (2020): Geschichte. Online: http://www.oecd.org/berlin/ dieoecd/ [28.02.2020].

Oschmiansky, F./Ebach, M. (2012): Vom AFG 1969 zur Instrumentenreform 2009: Der Wandel des arbeitsmarktpolitischen Instrumentariums. In: Bothfeld, S./Sesselmeier, W./Bogedan, C. (Hg.): Arbeitsmarktpolitik in der sozialen Marktwirtschaft. Vom Arbeitsförderungsgesetz zum Sozialgesetzbuch II und III. 2. überarbeitete Aufl. Wiesbaden: Springer VS, 91-105.
Oschmiansky, F./Mauer, A./Schulze Buschoff, K. (2007): Arbeitsmarktreformen in Deutschland - Zwischen Pfadabhängigkeit und Paradigmenwechsel. WSI-Mitteilungen, 60 (6), 2912-97.

Ostheim, T./Schmidt, M. G. (2007a): Vom Ausbau zur Konsolidierung: Sozialpolitik von der sozialliberalen Koalition bis zur Wiedervereinigung. In: Schmidt, M./Ostheim T./ Siegel, N.A./Zohlnhöfer, R. (Hg.): Der Wohlfahrtsstaat. Eine Einführung in den historischen und internationalen Vergleich. Wiesbaden: Springer VS, 165-173.

Ostheim, T./Schmidt, M. G. (2007b): Sozialpolitik nach der Wiedervereinigung. In: Schmidt, M./Ostheim T./Siegel, N.A./Zohlnhöfer, R. (Hg.): Der Wohlfahrtsstaat. Eine Einführung in den historischen und internationalen Vergleich. Wiesbaden: Springer VS, 193-209.

Rosenthal, P. (2012): Der Wandel der Arbeitslosenversicherung: Selektivität, Einkommenssicherung und Reziprozitätsnormen von 1969 bis heute. In: Bothfeld, S./Sesselmeier, W./Bogedan, C. (Hg.): Arbeitsmarktpolitik in der sozialen Marktwirtschaft. Vom Arbeitsförderungsgesetz zum Sozialgesetzbuch II und III. 2. überarbeitete Aufl. Wiesbaden: Springer VS, 143-190.

Schmid, G. (1987): Arbeitsmarktpolitik im Wandel: Entwicklungstendenzen des Arbeitsmarktes und Wirksamkeit der Arbeitsmarktpolitik in der Bundesrepublik Deutschland. WZB Discussion Paper IIM LMP 87-17. Wissenschaftszentrum Berlin für Sozialforschung (WZB), Berlin.

Schmid, G./Wiebe, N. (1999): Die Politik der Vollbeschäftigung im Wandel. Von der passiven zur interaktiven Arbeitsmarktpolitik. In: Kaase, M./Schmid, G. (Hg.): Eine lernende Demokratie. 50 Jahre Bundesrepublik Deutschland. Berlin: Edition Sigma, 357-396.

Schmidt, M. G. (1998): Sozialpolitik in Deutschland. Historische Entwicklung und internationaler Vergleich. Opladen: Leske \& Budrich.

Sell, S. (1998): Entwicklung und Reform des Arbeitsförderungsgesetzes als Anpassung des Sozialrechts an flexible Erwerbsformen? Mitteilungen aus Arbeitsmarkt und Berufsforschung, 31 (3), 432-548.

- (2000): Arbeitsmarktpolitik - Vom langsamen Sterben einer guten Absicht oder Renaissance im Modell des , aktivierenden Sozialstaates"? In: Schulz-Nieswandt, F. (Hg.): Sozialpolitische Trends in Deutschland in den letzten drei Dekaden. Berlin: Duncker und Humblot, 143-165.

Sesselmeier, W. (2017): Politikberatung in der Arbeitsmarktpolitik. In: Falk, S. (Hg.): Handbuch Politikberatung. Wiesbaden: Springer, 303-316.

Social Progress Imperative (2018): 2018 Social Progress Index. Executive Summary. Online: https://www.socialprogress. org/resources?filter $=2018$ [29.01.2019].

Statista (2019): Armut in Deutschland. Online: https:// de.statista.com/statistik/studie/id/50108/dokument/ armut-in-deutschland/ [14.08.2019].

Statistisches Bundesamt (Hg.) (2001): Datenreport 1999. Zahlen und Fakten über die Bundesrepublik Deutschland. Bonn: Bundeszentrale für politische Bildung. 
Steffen, J. (2019): Sozialpolitische Chronik. Online: http:// www.portal-sozialpolitik.de/sozialpolitische-chronik [10.03.2019].

Steiner, V./Hagen, T. (2001): Erfahrungen aus der Evaluation der Aktiven Arbeitsmarktpolitik in Deutschland. In: Steiner, V./Wolff, H. (Hg.): Mismatch am Arbeitsmarkt Was leistet die Arbeitsmarktpolitik? Ein Werktstattbericht zur Evaluation der Arbeitsmarktpolitik. Münster/New York/München/Berlin: Waxmann, 143-158.

Weishaupt, J. T. (2011): From the Manpower Revolution to the Activation Paradigm: Explaining Institutional Continuity and Change in an Integrating Europe. Amsterdam: Amsterdam University Press.

World Bank (2019): World Development Report 2019. THE CHANGING NATURE OF WORK. Washington DC: World Bank

WSI (2016): Befristete Beschäftigung nach Altersgruppen 1991-2016. Online: https://www.boeckler.de/5350o.htm [11.07.2019].

Zimmermann, G. E. (2001): Armut. In: Schäfers, B./Zapf, W. (Hg.): Handwörterbuch zur Gesellschaft Deutschlands. Wiesbaden: VS Verlag für Sozialwissenschaften, 36-52. Im Anhang:

Abbildung A1: Leistungsbeziehende und Leistungsquoten in der Arbeitslosen- und Grundsicherung

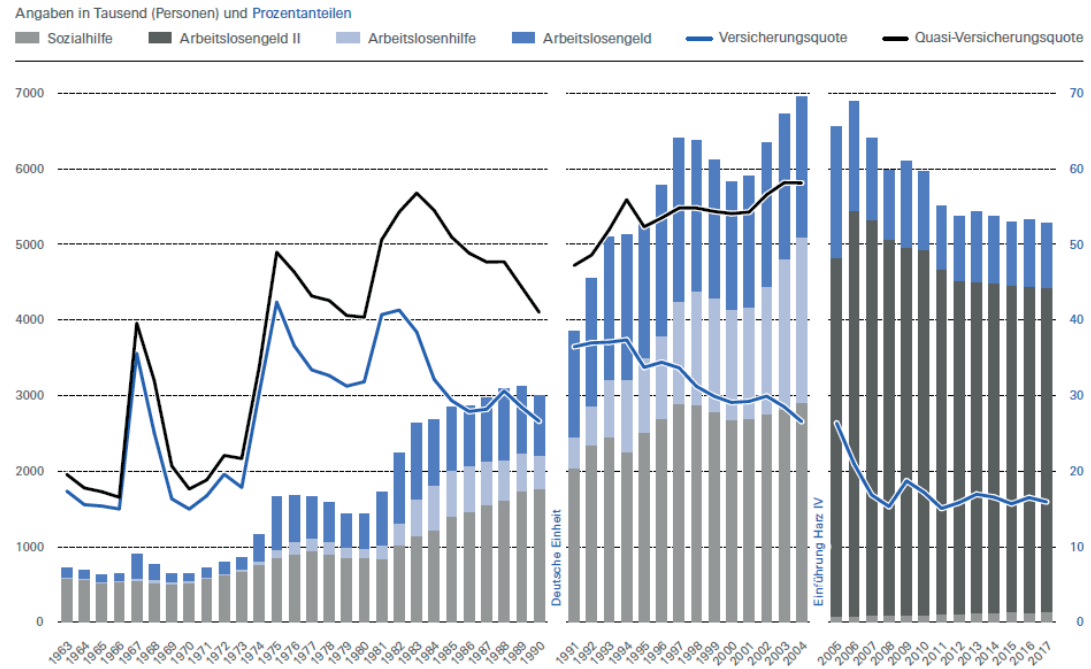

Quelle: Knuth 2018, aktualisiert durch Autor.

Abbildung A2: Teilnehmer in ausgewählten arbeitsmarktpolitischen Instrumenten 2006-2018 (Bestand im Jahresdurchschnitt)

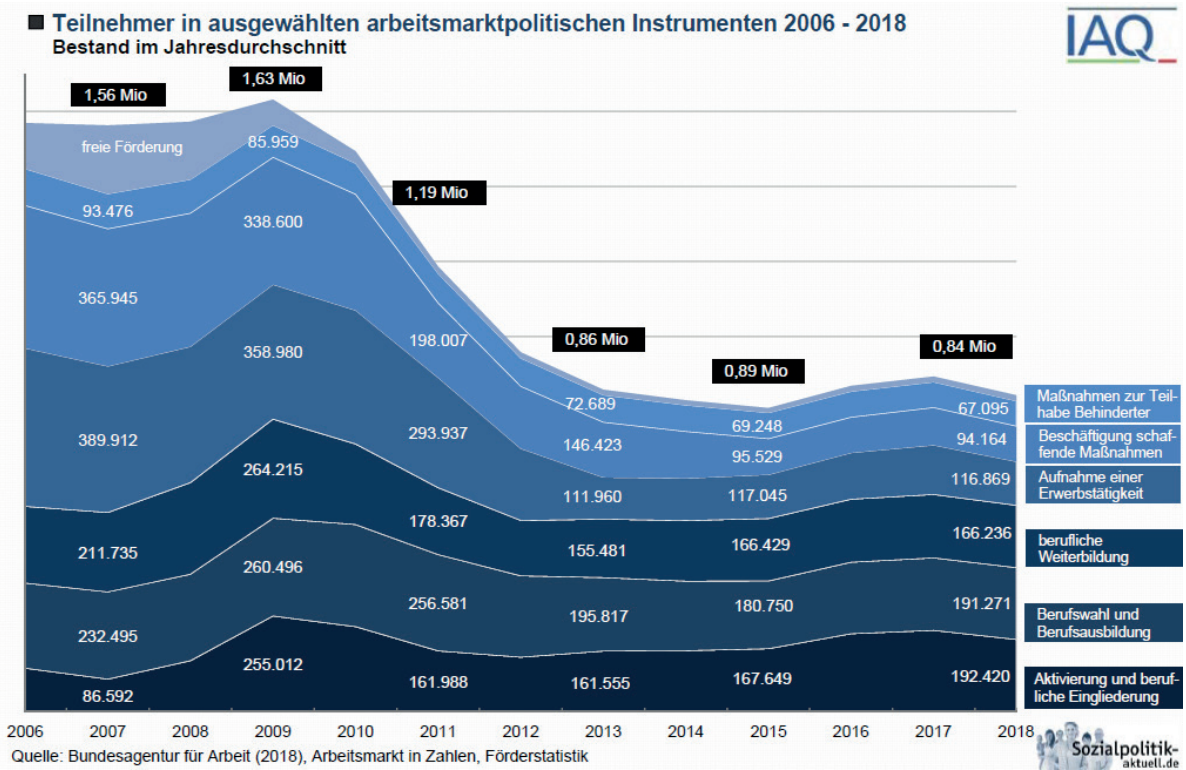

Quelle: Bundesagentur für Arbeit (2018), www.sozialpolitik-aktuell.de 